\title{
193.
}

\section{ON RODRIGUES' METHOD FOR THE ATTRACTION OF ELLIPSOIDS.}

[From the Quarterly Mathematical Journal, vol. II. (1858), pp. 333-337.]

THE following is in substance the method given in the "Mémoire sur l'attraction des Spheroïdes," par M. Rodrigues, Corresp. sur l'École Polyt., t. III. pp. 361-385 (1815). It will be seen that the method is very similar to that given two years before by Gauss, see my paper "On Gauss' Method for the Attraction of Ellipsoids," Journal, t. I. pp. 162-166 [164] : the solution in fact depends upon the geometrical theorem therein quoted, viz. if $M$ be any point, $P$ a point of a closed surface, $P Q$ the normal (lying outside the surface) at the point $P, d S$ the element of the surface at that point, and if $M Q$ denotes the angle $M P Q$ and $\overline{M P}$ the distance of the points $M$ and $P$, then, theorem, the integral

$$
\iint \frac{d S \cos M Q}{\overline{M P^{2}}}
$$

has for its value

$$
0,-2 \pi \text { or }-4 \pi
$$

according as $M$ is exterior to, upon, or interior to the surface.

Suppose that $M$ is the attracted point and taking $A, B, C$ for the semiaxes of the surface of the attracting ellipsoid, or, if we please, for any semiaxes of an arbitrary ellipsoidal surface confocal with the surface of the attracting ellipsoid, let $P$ be a point on the surface of the interior similar ellipsoid whose semiaxes are $r A, r B, r C$. The coordinates of $M$ are taken to be $a, b, c$, and those of $P$ are taken to be $x, y, z$, and the value of the potential is

$$
V=\int \frac{d m}{\overline{M P}},
$$

where $d m$ is the element of mass. 
We may write

$$
\begin{aligned}
& x=r A \xi, \\
& y=r B \eta, \\
& z=r C \zeta,
\end{aligned}
$$

and then $\xi, \eta, \zeta$ will be the coordinates of a point $P^{\prime}$ on the surface of a sphere, radius unity, corresponding in a definite manner to the point $P$ on the surface of the internal similar ellipsoid. And if $d \sigma$ be the element of the spherical surface, then we have

$$
d m=A B C r^{2} d r d \sigma
$$

and therefore

$$
\frac{V}{A B C}=\int \frac{r^{2} d r d \sigma}{\bar{M} \bar{P}}
$$

where, in order to obtain the value of the potential $V$ for the ellipsoid whose semiaxes are $A, B, C$, the integrations must be extended over the spherical surface and from $r=0$ to $r=1$.

Suppose that $d S$ is the element of the internal similar surface at $P$, and let $p$ be the perpendicular from the centre upon the tangent plane at $P$, we have

$$
d S=\frac{r^{3} A B C}{p} d \sigma
$$

Let $P_{0}$ be the point on the ellipsoid $(A, B, C)$ similarly situated to the point $P$ on the ellipsoid $(r A, r B, r C)$; the coordinates of $P_{0}$ are $A \xi, B \eta, C \zeta$; and if $p_{0}$ be the perpendicular from the centre upon the tangent plane at $P_{0}$, then $p=r p_{0}$, and the preceding equation becomes

$$
d S=\frac{r^{2} A B C}{p_{0}} d \sigma
$$

Imagine now an ellipsoidal surface confocal with the surface $(A, B, C)$ and having for its semiaxes

$$
A+\delta A, \quad B+\delta B, \quad C+\delta C,
$$

and let $P_{0}^{\prime}$ be the point on this surface which corresponds with the point $P_{0}$ on the surface $(A, B, C)$; that is, let $P_{0}^{\prime}$ be the point whose coordinates are

$$
(A+\delta A) \xi, \quad(B+\delta B) \eta, \quad(C+\delta C) \zeta
$$

and let $P^{\prime}$ be in like manner the point whose coordinates are

$$
r(A+\delta A) \xi, \quad r(B+\delta B) \eta, \quad r(C+\delta C) \zeta
$$


the points $P, P^{\prime}$ will be in like manner corresponding points on the surface $(r A, r B, r C)$ and on the confocal surface $\{r(A+\delta A), r(B+\delta B), r(C+\delta C)\}$; and if the normal distance at the point $P_{0}$ of the first two surfaces is $\delta N$, then the normal distance at the point $P$ of the second two surfaces will be $r \delta N$. The decrement of $\overline{M P}$ will be equal to the normal distance $r \delta N$ of the two surfaces at the point $P$ multiplied into the cosine of the angle $M Q$, and we have, by a property of confocal surfaces,

$$
A \delta A=B \delta B=C \delta C=p_{0} \delta N=(\text { suppose }) \frac{1}{2} \delta \theta
$$

we have therefore

$$
d \overline{M P}=-\frac{\frac{1}{2} r \delta \theta}{p_{0}} \cos M Q
$$

Hence from the equation

$$
\frac{V}{A B C}=\int \frac{r^{2} d r d \sigma}{\overline{M P}}
$$

We deduce

$$
\delta \frac{V}{A B C}=\int r^{2} d r d \sigma \frac{\frac{1}{2} r \delta \theta}{P_{0}} \frac{\cos M Q}{\overline{M P^{2}}} .
$$

But we have

$$
\frac{r^{2} d \sigma}{p_{0}}=\frac{d S}{A B C}
$$

and the equation thus becomes

$$
\delta \frac{V}{A B C}=\frac{\frac{1}{2} \delta \theta}{A B C} \int r d r \frac{d S \cos M Q}{\overline{M P^{2}}} .
$$

It may be proper to remark here by way of recapitulation that the course of the investigation has been as follows: viz. that, with a view to obtaining the potential $V$ of an attracting ellipsoid, we have found the increment of $\frac{V}{A B C}$ in passing from the ellipsoidal surface $(A, B, C)$ to the ellipsoidal surface $(A+\delta A, B+\delta B, C+\delta C)$, each of them confocal with the surface of the attracting ellipsoid; and that for finding such increment we have had to consider the two surfaces $(r A, r B, r C)$ and $\{r(A+\delta A)$ $r(B+\delta B), r(C+\delta C)\}$ confocal to each other and respectively similar to the firstmentioned two surfaces.

Resuming the formula just obtained, the integral with respect to $d S$ is taken over the entire surface of the internal similar ellipsoid $(r A, r B, r C)$, and if the attracted point $M$ is external to the ellipsoid $(A, B, C)$ it will be external to the interior similar ellipsoid $(r A, r B, r C)$ : hence in this case the double integral vanishes for all values of $r$, or we have

$$
\delta \frac{V}{A B C}=0 ;
$$


that is the function $V \div A B C$, which represents the ratio of the potential to the mass, is not altered in passing from the ellipsoid $(A, B, C)$ to the confocal ellipsoid

$$
(A+\delta A, \quad B+\delta B, \quad C+\delta C),
$$

or, what is the same thing, the potentials (and therefore the attractions) of confocal ellipsoids upon the same external point are proportional to their masses; this is in fact Maclaurin's theorem for the attraction of ellipsoids upon an external point.

But if the attracted point $M$ is interior to the ellipsoid $(A, B, C)$, then writing

$$
\frac{a^{2}}{A^{2}}+\frac{b^{2}}{B^{2}}+\frac{c^{2}}{C^{2}}=r^{\prime 2}
$$

where $r^{\prime}$ is less than unity, the double integral is $=0$ from $r=0$ to $r=r^{\prime}$ and is $=-4 \pi$ from $r=r^{\prime}$ to $r=1$, and we have

$$
\begin{aligned}
\delta \frac{V}{A B C} & =-\frac{2 \pi \delta \theta}{A B C} \int_{r^{\prime}}^{1} r d r \\
& =-\frac{\pi \delta \theta}{A B C^{\prime}}\left(1-r^{\prime 2}\right) \\
& =\frac{\pi \delta \theta}{A B C}\left(\frac{a^{2}}{A^{2}}+\frac{b^{2}}{B^{2}}+\frac{c^{2}}{C^{2}}-1\right) ;
\end{aligned}
$$

that is, the right-hand side of the equation is the increment (or taken with its sign reversed so as to be positive, it is the decrement) of the function $V \div A B C$ in passing from the ellipsoid $(A, B, C)$ to the confocal ellipsoid

$$
(A+\delta A, \quad B+\delta B, \quad C+\delta C)
$$

where

$$
\frac{1}{2} \delta \theta=A \delta A=B \delta B=C \delta C .
$$

The preceding formula gives at once the potential for an interior point; in fact taking $\alpha, \beta, \gamma$ for the semiaxes of the ellipsoid and writing

$$
A^{2}=\alpha^{2}+\theta, \quad B^{2}=\beta^{2}+\theta, \quad C^{2}=\gamma^{2}+\theta,
$$

and using the ordinary symbol $d$ instead of $\delta$, we have

$$
\frac{d}{d \theta} \frac{V}{\sqrt{ }\left\{\left(\alpha^{2}+\theta\right)\left(\beta^{2}+\theta\right)\left(\gamma^{2}+\theta\right)\right\}}=\frac{\pi}{\sqrt{ }\left\{\left(\alpha^{2}+\theta\right)\left(\beta^{2}+\theta\right)\left(\gamma^{2}+\theta\right)\right\}}\left\{\frac{a^{2}}{\alpha^{2}+\theta}+\frac{b^{2}}{\beta^{2}+\theta}+\frac{c^{2}}{\gamma^{2}+\theta}-1\right\},
$$

and integrating from $\theta=0$ to $\theta=\infty$, we have

$$
-\frac{V}{\alpha \beta \gamma}=\pi \int_{0}^{\infty} \frac{d \theta}{\sqrt{ }\left\{\left(\alpha^{2}+\theta\right)\left(\beta^{2}+\theta\right)\left(\gamma^{2}+\theta\right)\right\}}\left\{\frac{a^{2}}{\alpha^{2}+\theta}+\frac{b^{2}}{\beta^{2}+\theta}+\frac{c^{2}}{\gamma^{2}+\theta}-1\right\},
$$


where $V$ is now the potential for the ellipsoid whose semiaxes are $\alpha, \beta, \gamma$; and we have therefore

$$
V=-\pi \alpha \beta \gamma \int_{0}^{\infty} \frac{d \theta}{\sqrt{ }\left\{\left(\alpha^{2}+\theta\right)\left(\beta^{2}+\theta\right)\left(\gamma^{2}+\theta\right)\right\}}\left\{\frac{a^{2}}{\alpha^{2}+\theta}+\frac{b^{2}}{\beta^{2}+\theta}+\frac{c^{2}}{\gamma^{2}+\theta}-1\right\} .
$$

To find the potential for an external point it is only necessary to remark that by the theorem above demonstrated $V \div \alpha \beta \gamma$ is equal to the corresponding function for the confocal ellipsoid through the attracted point, that is for the ellipsoid whose semiaxes are $\sqrt{ }\left(\alpha^{2}+\theta_{1}\right), \sqrt{ }\left(\beta^{2}+\theta_{1}\right), \sqrt{ }\left(\gamma^{2}+\theta_{1}\right)$, where $\theta_{1}$ is a positive quantity such that

$$
\frac{a^{2}}{\alpha^{2}+\theta_{1}}+\frac{b^{2}}{\beta^{2}+\theta_{1}}+\frac{c^{2}}{\gamma^{2}+\theta_{1}}=1
$$

hence in the value of $V \div \alpha \beta \gamma$ we have only to write the above values in the place of $\alpha, \beta, \gamma$; and if we then write $\theta-\theta_{1}$ in the place of $\theta$ the limits will be $\infty, \theta_{1}$, and the expression for the potential is

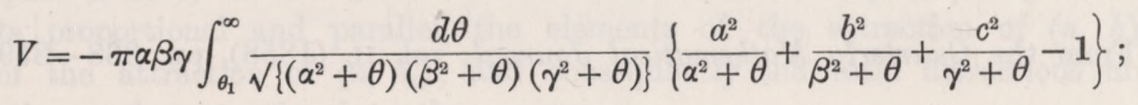

this completes the investigation. 